\title{
Coupled air/granular flow in a linear Hele-Shaw cell
}

\author{
Ø. Johnsen, ${ }^{1}$ R. Toussaint, ${ }^{2}$ K. J. Måløy, ${ }^{1}$ E. G. Flekkøy, ${ }^{1}$ and J. Schmittbuhl ${ }^{2}$ \\ ${ }^{1}$ Department of Physics, University of Oslo, P.O. Box 1048, Blindern, 0316 Oslo, Norway \\ ${ }^{2}$ IPGS, Université Louis Pasteur, 5 rue René Descartes, 67084 Strasbourg Cedex, France
}

(Received 16 April 2007; published 7 January 2008)

\begin{abstract}
We investigate experimentally the pattern formation process during injection of air in a noncohesive granular material confined in a linear Hele-Shaw cell. We characterize the features and dynamics of this pattern formation on the basis of fast image analysis and sensitive pressure measurements. Behaviors are classified using two parameters — injection pressure and plate opening — and four hydrodynamic regimes are defined. For some regions of the parameter space, flows of air and grains are shown to be strongly coupled and instable, and lead to channelization within the granular material with obvious large-scale permeability variations.
\end{abstract}

DOI: 10.1103/PhysRevE.77.011301

PACS number(s): 45.70.-n, 83.50.-v, 47.15.gp

\section{INTRODUCTION}

During air injection into noncohesive and weakly confined granular materials where particles are able to move together with the fluid phase, a variety of patterns are formed. In particular when the particles are small, the gasgrain interactions are of central importance. Such interactions are host to a wide range of fascinating physical phenomena and play a vital role in a variety of systems from fluidized beds [1-4] to flow in tubes [5-10] and ticking hour glasses $[11,12]$.

Instabilities during air injection into confined nonconsolidated granular materials has previously been studied for a radial geometry $[13,14]$. It is found that the obtained patterns can be classified into different hydrodynamical regimes depending on the injection pressure. Similarly characteristic flow regimes are studied when a fluid is retracted from a nonconsolidated porous medium $[15,16]$.

The patterns and instabilities observed in such experiments bear resemblance to Hele-Shaw [17] experiments where viscous fluid invades a more viscous one, either using Newtonian [18-21] or non-Newtonian fluids [22-25]. For the non-Newtonian case a transition from a viscous fingering regime to a viscoelastic fracturing regime is observed [26-28]. Multiphase flows in immobile porous media are other systems with general similarities [29-49].

Studies of coupled fluid/granular flow can be of great geological interest and provide insight into underlying physical processes taking place in, for instance, fluid intrusions into sediment rocks and hydrofracture [50-54]. Viscoelastic fracturing and hydrofracture have also been subject to several numerical studies [55-58].

Similarly to the Saffman-Taylor instability [18] observed in nonmiscible biphasic flows, the driving force of the granular motion is the fluid pressure gradient. Given the fact that the pressure is more or less constant inside the grainless region, the pressure gradient being the largest at the tip [59], the particles near the finger tip experience a higher drag force in the flow direction. This favors the growth of the most advanced finger while the growth of shorter fingers is suppressed. On the other hand, an important difference from the classical Saffman-Taylor problem is that in the granular case surface tension is absent. The stabilizing mechanisms are a matter of friction mobilization between the granular matrix and the confining plates rather than surface tension as in the classical two-fluid system. The structures studied in the dry granular case do, in other words, depend on the existence of friction.

Phase mixing is also an essential difference between the coupled fluid/granular system and nonmiscible biphasic flows, such as, e.g., the viscoelastic biphasic flow and the Saffman-Taylor instability between two fluids. In many systems the phases are separate, i.e., nonmiscible, while for our granular configuration the phases are interpenetrating. The phases can be defined from a mesoscopic point of view by their particle density: distinguishing between a fluid phase where this density is low and a powder phase where it is higher. Both states exchange fluid, i.e., interpenetrate (even though at smaller scales, one solid grain does not exchange mass with the surrounding fluid). Since the phases are able to mix, the driving force-i.e., the pressure gradient acting upon the displacement front-will dissipate in time. This mechanism also contributes to stabilization of the pattern formation.

In this study, we characterize and quantify the global features of pattern formations and their associated dynamic processes during injection of air in a noncohesive granular material confined in a linear Hele-Shaw cell. This geometry is complementary to the circular cell used in Refs. $[13,14]$, and these two represent the standard geometries for studying flow problems such as, e.g., the two-fluid Saffman-Taylor instability or multiphase flows in porous media. The linear cell geometry allows us to study the translation symmetry breaking during destabilization since it is spatially invariant along the flow direction, while the rotational symmetry breaking has previously been investigated in the radially invariant circular cell $[13,14]$. Using a fast digital camera, we obtain a quantitative description of the patterns and their evolution. We explore the parameter space using three different openings and a large range of injection pressure.

The article is organized as follows: In Sec. II the experimental setup, sample preparations, and imaging methods are described. In Sec. III we present our experimental results: we describe the observed hydrodynamical regimes and classify the different pattern formed into a phase diagram as function of the pressure and plate separation. Section IV is dedicated to characterizing the geometrical and dynamical features of 


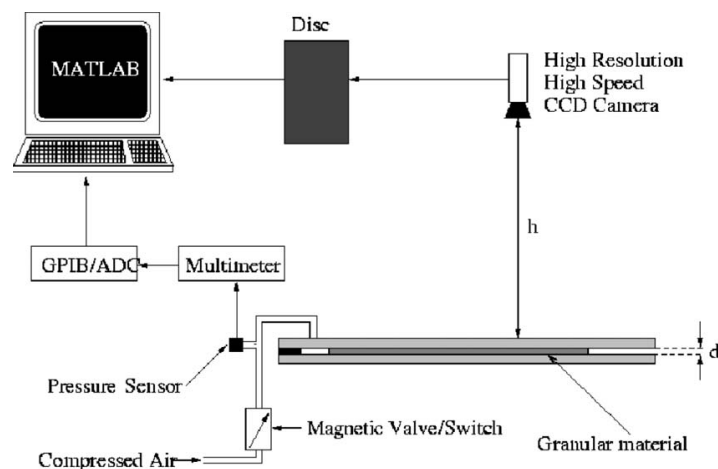

(a)

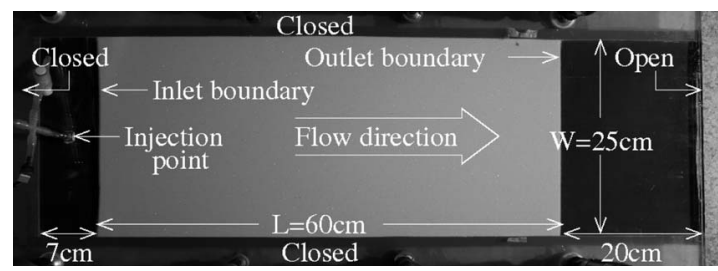

(b)

FIG. 1. (a) Experimental setup. The horizontally fixed HeleShaw cell is connected to a pressure-control unit and air is injected at constant overpressure when the magnetic valve is triggered to open. A high-speed, high-resolution charge-coupled-device (CCD) camera is used to follow the development of the displacement pattern and pressure is read at the inlet. (b) Image of the $25 \mathrm{~cm}$ $\times 87 \mathrm{~cm}$ channel before the experiment begins. The cell is open in the direction of the indicated flow direction and closed elsewhere. During the experiment, air is injected through the tube near the closed boundary on the left opposite to the outlet. The cell is filled with approximately $25 \mathrm{~cm} \times 60 \mathrm{~cm} \times d$ of loosely packed material, consisting of polydisperse spherical glass particles, with mass density $\rho=2.6 \mathrm{~g} \mathrm{~cm}^{-3}$ and diameters distributed between 75 and $150 \mu \mathrm{m} . d$ is the plate separations used: $0.7 \mathrm{~mm}, 1.1 \mathrm{~mm}$, or 2.1 $\mathrm{mm}$. In order to apply the injected pressure, hence the pressure gradient, over the whole straight front and not only at a point just immediately near the injection hole, there is a $\sim 5 \mathrm{~cm} \times 25 \mathrm{~cm}$ inlet chamber free of particles on the left around the injection point.

the system. In Sec. V we present a theoretical model and compare its predictions to experiments before concluding and summarizing in Sec. VI.

\section{SYSTEM DESCRIPTION}

\section{A. Experimental setup}

Figure 1(a) shows a simple sketch of the experimental setup, and Fig. 1(b) shows the sample before air injection. A Hele-Shaw cell [17] is built from two 1-cm-thick glass plates with dimensions $40 \mathrm{~cm} \times 90 \mathrm{~cm}$. One of the short boundaries and both of the long ones are sealed with a silicon rubber band and double-sided tape and then clamped together. These are labeled "Closed" in Fig. 1(b). The other short boundary, on the right-hand side in Fig. 1(b), is open. This gives a $25 \mathrm{~cm} \times 87 \mathrm{~cm} \times d$ channel. The injection hole is $4 \mathrm{~mm}$ in diameter and is situated $3 \mathrm{~cm}$ from the sealed short boundary along the longitudinal center. The system is studied for three different separations between the plates; $d$ $=0.7 \mathrm{~mm}, d=1.1 \mathrm{~mm}$, and $d=2.1 \mathrm{~mm}$. Measuring the thickness of the glass plates individually and in the cell configuration at several locations, the plates are ensured to be parallel prior to performing the experiments.

The cell with a ready made sample is placed horizontally and connected to a gravitationally driven pressure source. At the inlet a pressure sensor is mounted in order to record the injection pressure. Images are acquired at a frame rate of 250 frames per second using a Redlake MotionPro high-speed charge-coupled-device (CCD) camera with spatial resolution of $1024 \times 1280$ pixels. Pixels have a size of $0.6 \mathrm{~mm}$, which corresponds to 4-8 particle diameters. The pressure injection is described by a step function in time: In the "low state," before air injection, the pressure recorded at the inlet equals the atmospheric pressure $P_{0}$. In the "high state," during air injection, the inlet pressure is maintained within a few percent at $P_{0}+\Delta P$, where $\Delta P$ is the imposed overpressure. The pressure step and image acquisition are synchronized. Injection of air into the model is triggered by switching on a magnetic valve $1 \mathrm{~s}$ after the beginning of the image sampling and the pressure recording.

\section{B. Sample preparations}

The granular material consists of polydisperse spherical glass beads, with diameters between 75 and $150 \mu \mathrm{m}$. Cohesion of a material consisting of beads of such small sizes is very sensitive to the humidity of the ambient air. To ensure the reproducibility of the experiment, it is important to control the humidity at a suitable level. The relative humidity in the room is therefore kept around 35\% using a humidifier/ dehumidifier.

During sample preparations the cell is filled from the open cell boundary when held upright with loosely packed granular material. For each experiment the volume of the cell that is filled is approximately $25 \mathrm{~cm}$ (width) $\times 60 \mathrm{~cm}$ (length) $\times d$ (thickness). The outlet is then temporarily sealed with a metal plate, which is slid roughly $20 \mathrm{~cm}$ deep into the open end of the channel. With the plate preventing particles from falling out, the cell is turned upside down a couple of times to produce a homogeneous and loose packing. The measured initial solid fractions for the three plate separations is $\rho_{s}^{\text {init }}$ $\approx 0.55 \pm 0.03$. The presented method of sample preparation is chosen out of reproducibility purposes: We consider it to be more easily controllable than producing a more densely packed initial state by, e.g., gently tapping or vibrating the filled cell.

To position the granular packing inside the cell with a reproducible solid fraction and obtain straight boundaries at a reproducible distance from the inlet and outlet, the final rotation is done such that the particles fall inside the vertically positioned cell-from the inlet side toward the outlet blocked by the metal plate. The friction acting on the particles from the confining plates is then polarized [60] in the opposite direction of fluid/particle flow indicated in Fig. 1(b). The cell is then finally put in a horizontal position and the metal plate is removed. The position of the $60-\mathrm{cm}-10 n g$ 
granular packing inside the cell is such that ahead of the grains, a region of $20 \mathrm{~cm}$ between the grain packing and the cell opening is free of particles. The grain/air boundary facing the outlet is denoted the outlet boundary. Behind the packing, far to the left in the figure, a $\sim 7 \mathrm{~cm}$ (length) $\times 25 \mathrm{~cm}$ (width) $\times d$ (thickness) grain-free zone is acting as a "pressure-leveling" chamber. This large inlet chamber will ensure that the granular material feels the pressure gradient over the whole width of the cell rather than in a localized point around the injection point as would be the case if we were to inject directly into the granular matrix. This will allow us to study the translation symmetry breaking during the destabilization of initially straight fronts. The rotational symmetry breaking in the case of point injection has been previously studied [14]. The grain/air boundary facing the inlet is called the inlet boundary.

\section{Image analysis}

Image analysis provides several pieces of quantitative information on the ongoing processes like boundary motions or compaction processes. Indeed, when air is injected at sufficient overpressure, a zone free of grains develops around the inlet. As this emptied structure grows, the material ahead of it is compacted over a depth dependent on the size of the structure. With sufficiently high spatial resolution, as provided by our recording equipment, the compacted zone can be determined by image analysis.

We load two images of interest, such as, for instance, Figs. 2(a) and 2(b), into two matrices in our MATLAB imageprocessing program. Subtracting the gray levels between these images, pixel by pixel, and thresholding the resulting subtraction image at a suitable gray level, we can both extract the emptied displacement pattern and detect the motion of grains ahead of the emptied zone. After subtraction and thresholding, we obtain the result shown in Fig. 2(c), where the emptied pattern appears as a solid white region, while the particle displacements within the packing itself appear as white speckles on a black background. For presentation purposes the speckles are dilated such that they are visible in an image of such small scale. The well-defined densely speckled region ahead of the emptied zone is the compacted region where particles have been rearranged internally in the packing. There may be some isolated speckles both outside the defined compacted region and outside the cell. These are not due to particle motion, but are regarded as noise due to light flickering and reflection over single glass beads. In order to have a well-defined way of recognizing the compacted zone, we separate the densely speckled zone from the isolated speckles by performing a coarse-graining spatial filter as follows: We find a suitable box size, typically $1 \mathrm{~cm}$, to check for speckle density. We move the box over the subtraction image and box-sized regions containing two or more speckles are regarded as belonging to the compaction region and tagged in gray. If there is only one or zero speckles within the reach of one box, the zone is regarded as immobile and tagged in black. The compaction region is found as the largest cluster and is shown in gray in Fig. 2(d), while the unperturbed region ahead appears all black. The vertical white

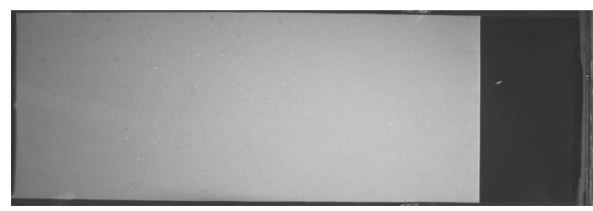

(a)

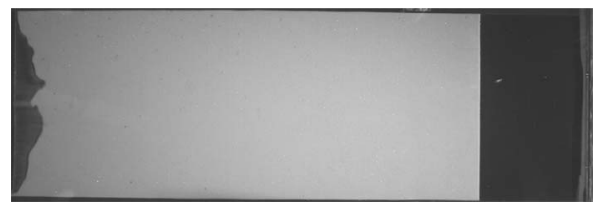

(b)

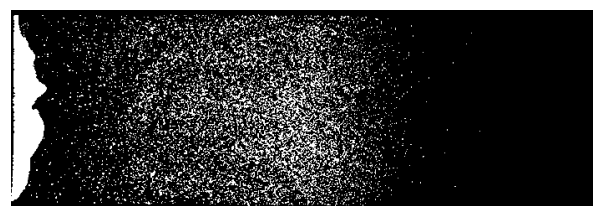

(c)

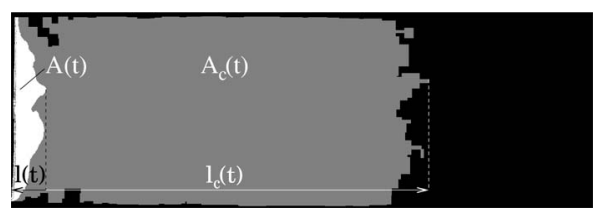

(d)

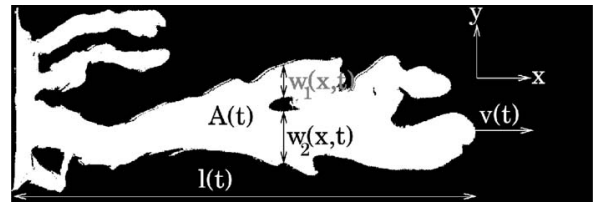

(e)

FIG. 2. Treating two images, one before injection (a) and one during and after (b), one can extract both the displacement structure and identify a speckled region ahead of it, representing a region where the granular material is compacted (c). Further image treatment allows us to extract the well-defined compacted region (d). (e) gives an example of the fingering growth phase and of the geometrical quantities that are typically measured.

line indicates the position of the outlet grain boundary.

This image treatment allows us to easily measure geometrical quantities for the emptied region, like, e.g., the emptied area $A(t)$, finger tip positions $l(t)$, and finger tip velocity $v(t)$ as indicated in Fig. 2(e). The finger width is defined as the total of the widths over all $n$ individual fingers crossing position $x$ at time $t: w(x, t)=\sum_{i=1}^{n} w_{i}(x, t)$, as shown in Fig. 2(e). For the compacted region, the area $A_{c}(t)$ and the linear extent $l_{c}(t)$ are also measured, as indicated in Fig. 2(d).

\section{FOUR PRESSURE REGIMES}

\section{A. Phenomenology}

In Fig. 3 we show and compare some of the experiments that we have conducted for the three different plate spacings at a range of injection pressures. The patterns and their dy- 


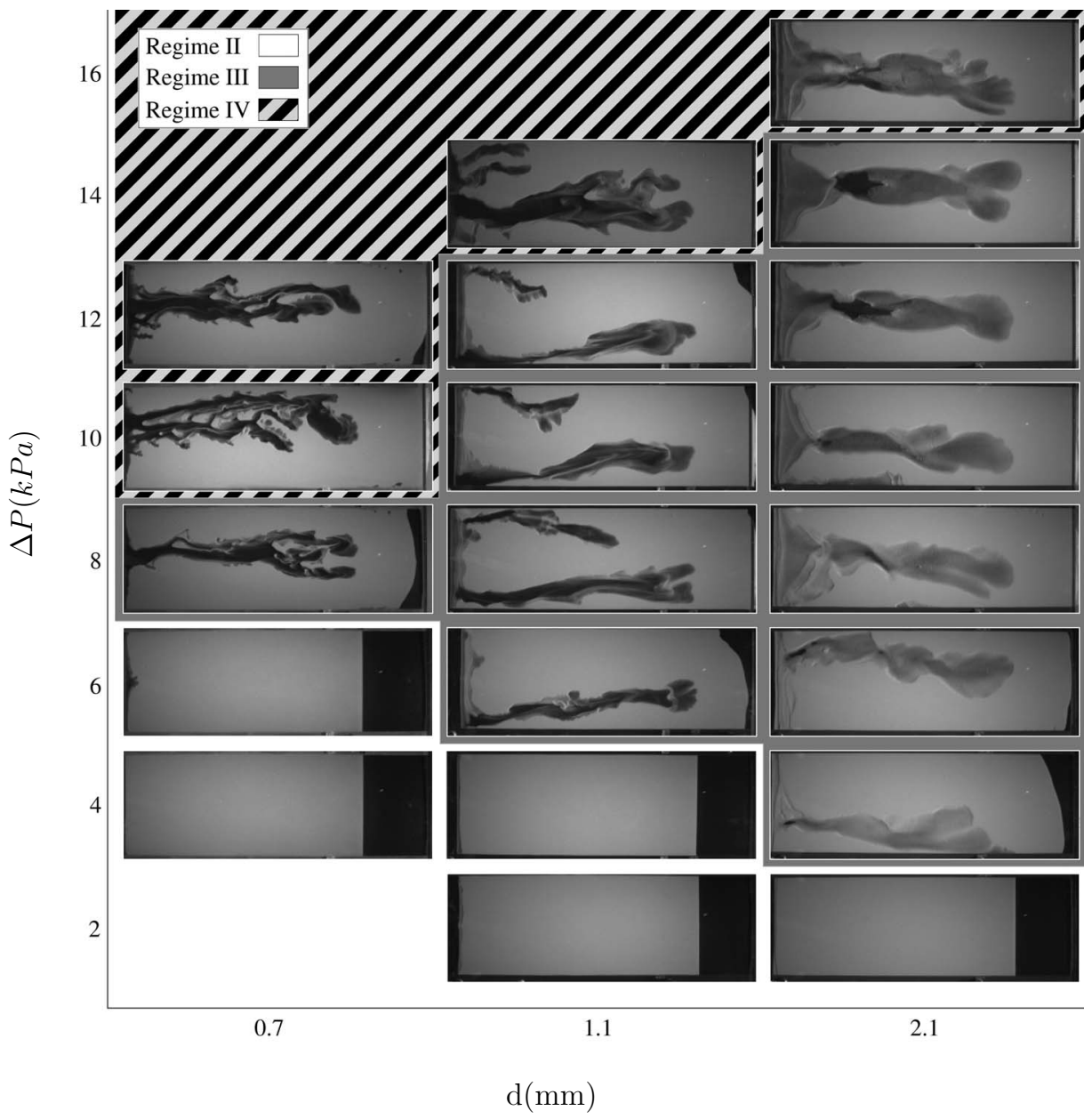

FIG. 3. Pattern formations for different pressures and plate separations. Apart from the experiment at $6 \mathrm{kPa}$ for the $0.7-\mathrm{mm}$ cell spacing, the experiments presented for regime II are attributed with displacements of short extent, and some particles are left inside the otherwise emptied structures. In these small-scale images this makes the displacements of these particular experiments not so easily visible.

namic properties are largely dependent on the pressure of the injected air, and the patterns formed can be classified into four different regimes as a function of this parameter.

\section{Regime I: $\Delta P<P_{1}$}

For injection pressures $\Delta P$ below some threshold pressure $P_{1}$ the hydrodynamic drag on the particles is not large enough to produce any detectable particle motion. The granular packing remains rigid while the air just permeates through the pore space, leaving no displacement pattern.

\section{Regime II: $\boldsymbol{P}_{1}<\Delta P<P_{2}$}

For injection pressures $\Delta P>P_{1}$, small displacement patterns develop and stabilize after a time $t_{1}$ [see Fig. 4(a)]. Typically, $t_{1} \sim 0.1 \mathrm{~s}$ and $l\left(t_{1}\right) \sim 2 \mathrm{~cm}$. Figure $4(\mathrm{~b})$ shows the temporal development of the most advanced point of the emptied structure for $\Delta P=4.4 \mathrm{kPa}$ and $1.1-\mathrm{mm}$ plate separation. Both $t_{1}$ and $l\left(t_{1}\right)$ are increasing with injection pressure. The front of the patterns appears as relatively smooth with only some smaller bulges along it. Typically the pattern is not completely emptied of grains, but a layer a couple of grain diameters deep remains, lying on the bottom plate, inside the otherwise emptied structure. The depth of this sedimentary layer decreases with the injection pressure and increases with the plate separation. For large pressures and especially for $0.7-\mathrm{mm}$ plate separation, the bottom plate is swept clean of grains inside the emptied zones.

As the emptied structure grows, the material ahead of it is compacted over a depth dependent on the extent of the size of this emptied zone. The compacted region is represented in gray in Fig. 4(a), and the temporal evolution is shown in Fig. 4(b). The size of both the empty zone and their associated compacted region increases with increasing pressure. By conservation of grain mass we may write

$$
\rho_{s}^{\text {init }}\left(A+A_{c}\right)=\rho_{s}^{c o m p} A_{c},
$$

where $\rho_{s}^{\text {init }}$ is the initial solid fraction, $\rho_{s}^{\text {comp }}$ the solid fraction of the compacted area, and $A$ and $A_{c}$ are, respectively, the area of the emptied structure and the compacted zone as defined in Fig. 2(d). Equation (1) gives the solid fraction of the compacted region, $\rho_{s}^{c o m p} \approx 0.58 \pm 0.03$, which is $\sim 5 \%$ denser than the initial solid fraction $\rho_{s}^{\text {init }}$.

Close to the upper transition pressure $P_{2}$, stick-slip motion of the front ahead of the empty structure is observed. The front advances one step and then stops again-often repeatedly. The outlet boundary is not observed to move except close to $P_{2}$, where it bulges slightly. For the 1.1-mm cell, deformation occurs for injection pressures above $5 \mathrm{kPa}$. From image subtraction it is seen that the deformation is localized in a zone smaller than the cell width and connected to the outer boundary, but disconnected from the inlet and 


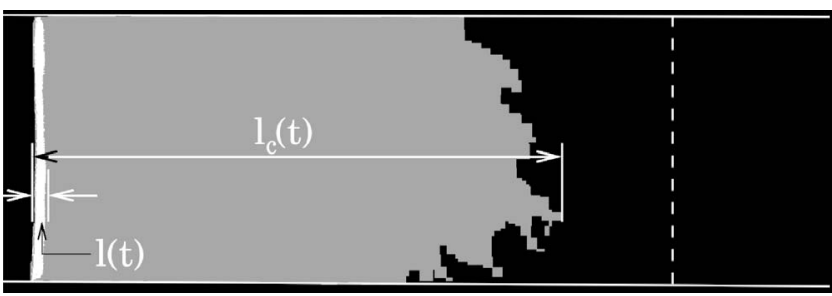

(a)

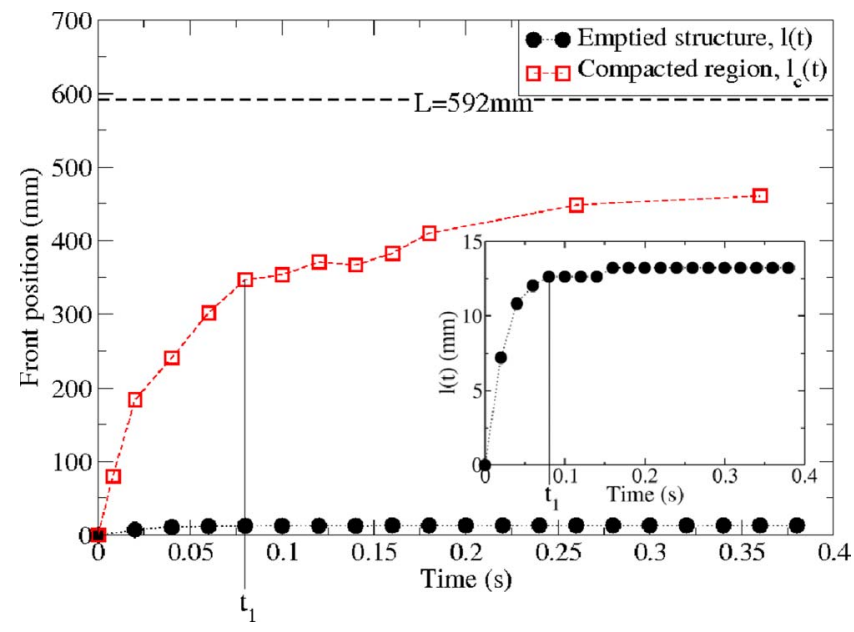

(b)

FIG. 4. (Color online) (a) Image subtraction between an image in the final state and a picture before injection of air reveals both the emptied structure (white) and compacted region (gray) for an experiment performed at injection pressure $\Delta P=4.4 \mathrm{kPa}$ (second pressure regime) and with $1.1-\mathrm{mm}$ plate separation. $l(t)$ and $l_{c}(t)$ are the most advanced points of, respectively, the emptied and compacted zones. The vertical white dashed line indicates the position of the granular packing's outlet grain boundary. (b) Temporal evolution of the most advanced point of the emptied structure $l(t)$, and the associated compacted region $l_{c}(t)$, in correspondence with (a). The inset in (b) shows the most advanced point of the emptied structure at a finer length scale. At time $t_{1}$, the empty structure stops evolving.

the compacted region ahead of it. Thus, we interpret this grain motion at the outlet as a result of the hydrodynamic drag felt by the outermost particles when the pressure gradient reaches a significant level. An alternative cause for this motion at the outer boundary would be a force resulting from the motion of the grains at the inner boundary, being transmitted through solid contacts from grain to grain through the whole packing. That would, however, result in a granular motion all along the grains, transmitting the force, which does not correspond to the observation of two disconnected regions where grains move, around the inlet and around the outlet. As indicated by the fact that the region of the mobile grains around the outlet expands in volume, the material is being locally decompacted starting at the outlet boundary. The boundary of this mobile region is a decompaction front which progresses in the direction opposite of the air and grain flow direction. In this second pressure regime, this motion is transient, and this front stops at a few centimeters from the outlet. While it is a transient feature in this regime, the decompaction starting at the outlet and propagating backward is a characteristic and well-marked feature of the third pressure regime, where it is easier to visualize (see Fig. 5).

We define the second pressure regime by the fact that the inlet boundary eventually stops completely and never reaches the system outlet. In terms of applied overpressure, the pressure limits of this regime are denoted $P_{1}<\Delta P<P_{2}$.

\section{Regime III: $P_{2}<\Delta P<P_{3}$}

Above $P_{2}$, a structure similar to those seen in the second regime forms and stabilizes after $t_{1}$ seconds at a length $l\left(t_{1}\right)$. As in the second pressure regime, both $t_{1}$ and $l\left(t_{1}\right)$ are increasing with pressure. After $t_{1}$ the growth of the emptied structure stops, and the velocity of the front is zero apart from some occasional stick-slip movement. However, contrarily to the second regime, after this transient rest period, the empty zone starts growing again and eventually breaks through the entire cell. This feature defines the third pressure regime.

The time interval of no growth is seen as a plateau in Fig. 5(d). Figure 5(b) shows the early stages of growth of the emptied structure and the compacted region at $\Delta P$ $=5.6 \mathrm{kPa}$ and $d=1.1 \mathrm{~mm}$ [see Fig. 5(a) for color code]. Just as $l\left(t_{1}\right)$ is larger in the third regime than in the second, so is the extent of its associated compacted region. The solid fraction of the compacted region, $\rho_{s}^{\text {comp }}$, is $\sim 5 \%$ higher than the initial $\rho_{s}^{\text {init }}$.

In Fig. 3 the emptied structure is observed to have some small branches, with radii of curvature comparable to the cell thickness, for the cell with $0.7-\mathrm{mm}$ plate separation, while for $1.1 \mathrm{~mm}$ and $2.1 \mathrm{~mm}$ the front is increasingly bulged, but not ramified; i.e., its local radius of curvature is of the order of the cell width, or larger.

After some time $t_{2}$ the outlet grain boundary is observed to deform due to local decompaction. Figures 5(b) and 5(d) show that the decompacted region extends farther and farther into the packing, its rear front propagating backward, opposite to the flow direction. When the decompacted and compacted regions merge, we are no longer able to distinguish them with image subtraction, but by eye we observe that the decompacted region grows farther into the packing (bubbles empty of grains sometimes nucleate in the mobile region in a zone initially around the outlet and expanding backward toward the inlet). As the material is further decompacted, the in-plane friction decreases. The fluid drag is able to overcome the mobilized friction that initially stabilized the growth of the emptied structure. The granular material becomes locally fluidized and a "river" of grain flow connecting the decompacted and emptied structure is observed, while the propagation of the empty structure front resumes. Following the track of the granular river where bubbles have sometimes appeared, the emptied zone becomes a propagating finger, which eventually breaks through the entire packing. Such a typical finger structure is shown in Fig. 5(c) and is formed after a characteristic time $t_{3}$ indicated in Fig. 5(d). During the propagation of the empty finger, the air flow erodes the surrounding grains and carries them along, eroding its side boundaries. Ahead of the growing finger the granular material is mobilized over the whole cell width. We 


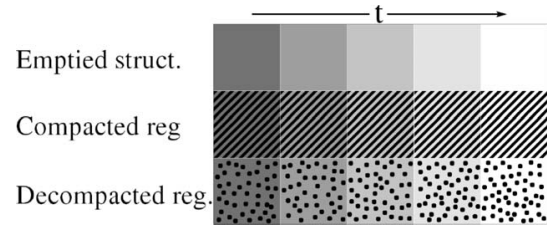

(a)

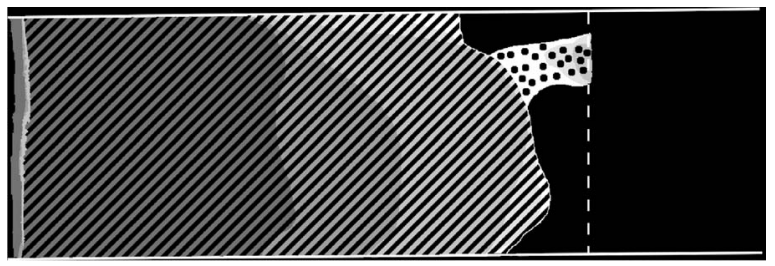

(b)

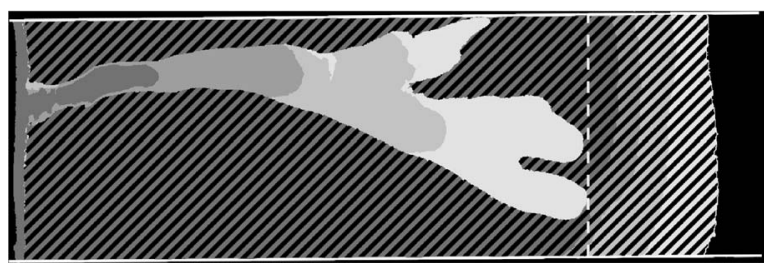

(c)

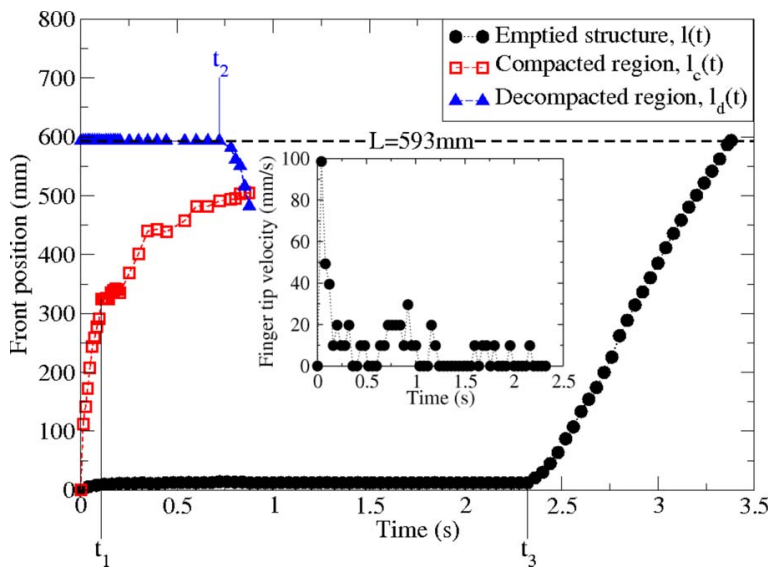

(d)

FIG. 5. (Color online) (a) Color coding of the emptied structure, compacted region, and decompacted region as function of time in (b) or emptied structure and mobile region in (c). (b) Early stages of the evolution of emptied, compacted and decompacted region $(t$ $=0.01 \mathrm{~s}, t=0.60 \mathrm{~s}, t=0.78 \mathrm{~s}, t=0.84 \mathrm{~s}$, and $t=0.90 \mathrm{~s})$ at pressure $\Delta P=5.6 \mathrm{kPa}$ (the third pressure regime) and plate separation $d$ $=1.1 \mathrm{~mm}$. The vertical dashed line indicates the initial position of the outlet grain boundary of the granular packing. (c) Fingering stage of the structure growth. The position of the finger tip is $l$ $=L / 4(t=2.60 \mathrm{~s}), l=L / 2(t=2.86 \mathrm{~s}), l=3 L / 4(t=3.09 \mathrm{~s})$, and $l$ $=L(t=3.37 \mathrm{~s})$, where $L=593 \mathrm{~mm}$ is the initial length of the packing indicated with a dashed line. The shaded zones correspond here to mobile grain regions. (d) Position of the most advanced point of the emptied structure $l(t)$, compacted zone $l_{c}(t)$, and decompacted zone $l_{d}(t)$ as a function of time. Onset of growth events $t_{1}, t_{2}$, and $t_{3}$ is indicated. The dashed line indicates the initial position of the outlet boundary. The inset shows the velocity profile of the early growth stage and the rest period. The small peaks indicates stickslip motion of the structure front. are not able to distinguish this zone of granular flow as being compacted or decompacted. Hence the hatched marked region in Fig. 5(c) refers to the zone where grains have moved during a time $t$.

When the finger has penetrated the granular packing, forming a wide open channel linking the injection point to the open boundary, the flow accelerates suddenly, and due to the limitations of the source pump, the overpressure within the emptied region can no longer be sustained. The drag is consequently reduced, and the rest of the structure will not be able to grow any further. When the monitored overpressure in the inlet chamber decreases more than a few percent, the subsequent stages are not taken into account in the analysis, since this last stage is device dependent, being associated with the limitations of the source pump.

For injection pressures close to the upper threshold of the third regime, the deformation of the outlet boundary is observed to be a result of the compacted region extending over the length of the packing, rather than the result of a fluid drag becoming significant at the outlet. This transition will be characterized more quantitatively in Sec. IV.

During the resting time $t_{3}-t_{1}$, from the moment when the empty zone stops growing until it starts again to develop toward breakthrough, the front of the emptied structure typically undergoes some stick-slip motion-growing an increment and stopping again, repeatedly. This is indicated in the inset plot of the velocity as a function of time for the early stage of growth and the rest state in Fig. 5(d).

$t_{3}-t_{1}$ is decreasing with increasing pressure and approaches zero near the transition pressure $P_{3}$. Injection pressures where no transient rest of the growth of the empty zone is observed mark the transition to the fourth pressure regime.

\section{Regime IV: $\Delta P>P_{3}$}

Exceeding $P_{3}$ the transient stage of no growth of the empty zone does not exist, and the finger formation will grow continuously to breakthrough, a feature defining the fourth pressure regime. Figures 6(a) and 6(b) display the temporal evolution for the emptied pattern formation and its associated compacted region for an experiment at $14 \mathrm{kPa}$ in a 1.1-mm-thick cell, respectively, at an early stage and during the fingering stage. Figure 6(c) shows the position of the emptied and compacted fronts. Within this pressure regime, the deformation of the outlet grain boundary is not due to decompaction, as will be shown in Sec. IV. The injection pressure is high enough to overcome jamming effects and pushes the granular material like a piston. In such a process the compacted region reaches and deforms the outlet boundary in $t_{2} \sim 0.2 \mathrm{~s}$. During compaction, the solid fraction increases by $\sim 5 \%-6 \%$ with respect to the initial one.

The advancing fingers, as shown in Fig. 6(b), is different from those in the third pressure regime in the sense that they are wider and more eroded. From Fig. 3 we also see that the influence of a reduction of the plate separations leads to more numerous and more complex fingers.

\section{B. Phase diagram}

The transition pressures between the regimes used for classifying the displacement patterns change with the plate 


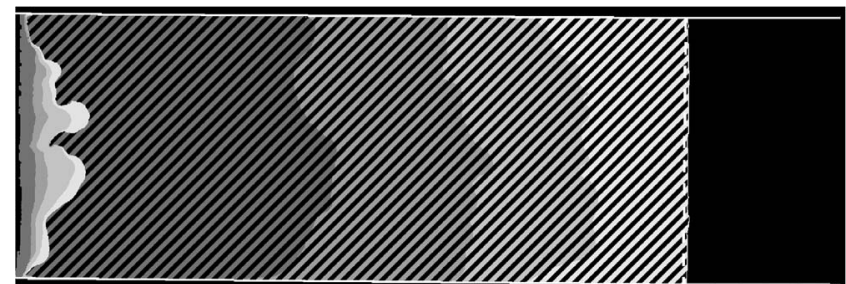

(a)

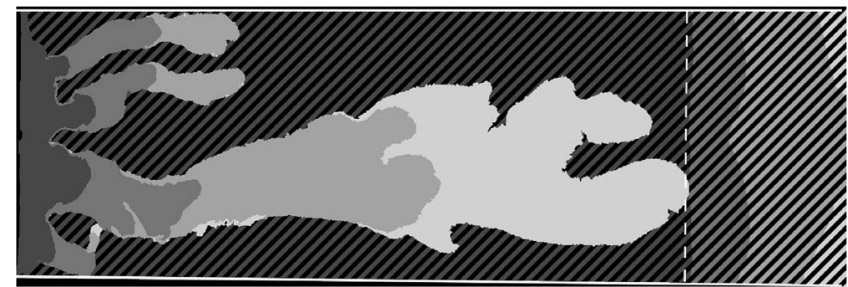

(b)

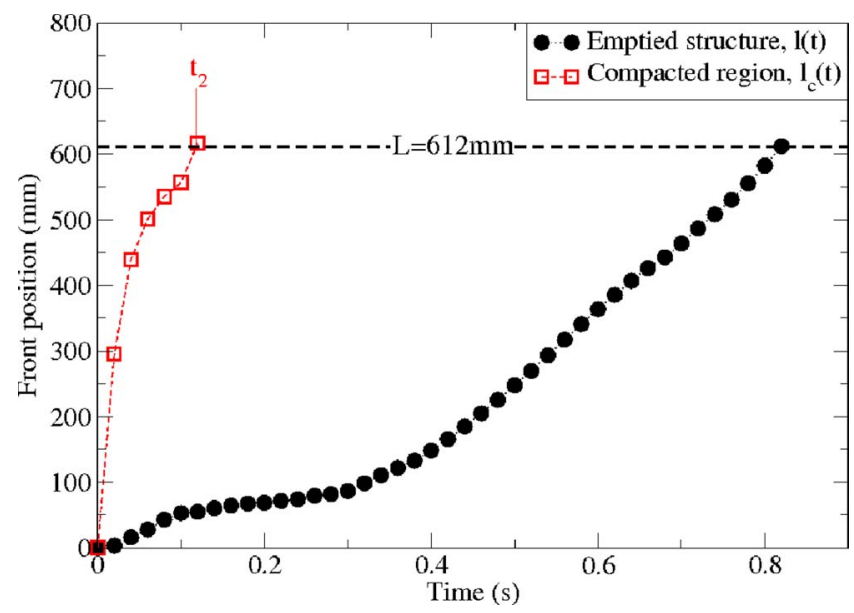

(c)

FIG. 6. (Color online) (a) Early stages of emptied and compacted regions with $\Delta P=14 \mathrm{kPa}$ and plate separation $d=1.1 \mathrm{~mm}$ (fourth pressure regime). The positions of the respective fronts are shown for $0.02 \mathrm{~s}, 0.04 \mathrm{~s}, 0.08 \mathrm{~s}, 0.16 \mathrm{~s}$, and $0.20 \mathrm{~s}$ following the same color code as Fig. 5(a). The vertical white line to the right indicates the outlet boundary of the granular packing $t=0$. (b) shows the pattern formation at a later stage; $t=0.2 \mathrm{~s}, 0.4 \mathrm{~s}, 0.6 \mathrm{~s}$, and $0.8 \mathrm{~s}$. In (c) the position of the most advanced point of the emptied zone, $l(t)$, and the compacted region, $l_{c}(t)$, is plotted versus time.

separation. In Fig. 3 experiments for plate spacings $0.7 \mathrm{~mm}$, $1.1 \mathrm{~mm}$, and $2.1 \mathrm{~mm}$ are displayed and compared for a range of injection pressures. This information can be condensed into and visualized in form of a phase diagram as in Fig. 7.

The minimum pressure required to mobilize particles defines the transition pressure $P_{1}$ between the first pressure regime and the second. Depending on the plate separation, we measure $P_{1}(0.7 \mathrm{~mm}) \simeq 0.20 \pm 0.05 \mathrm{kPa}, P_{1}(1.1 \mathrm{~mm})$ $\simeq 0.25 \pm 0.05 \mathrm{kPa}$, and $P_{1}(2.1 \mathrm{~mm}) \simeq 0.35 \pm 0.1 \mathrm{kPa}$. The transition from the second pressure regime to the third is taking place at a lower pressure as the plate separation is increased; i.e., $P_{2}$ is a decreasing function of the plate separation: $P_{2}(0.7 \mathrm{~mm}) \simeq 6.3 \pm 0.5 \mathrm{kPa}, P_{2}(1.1 \mathrm{~mm})$

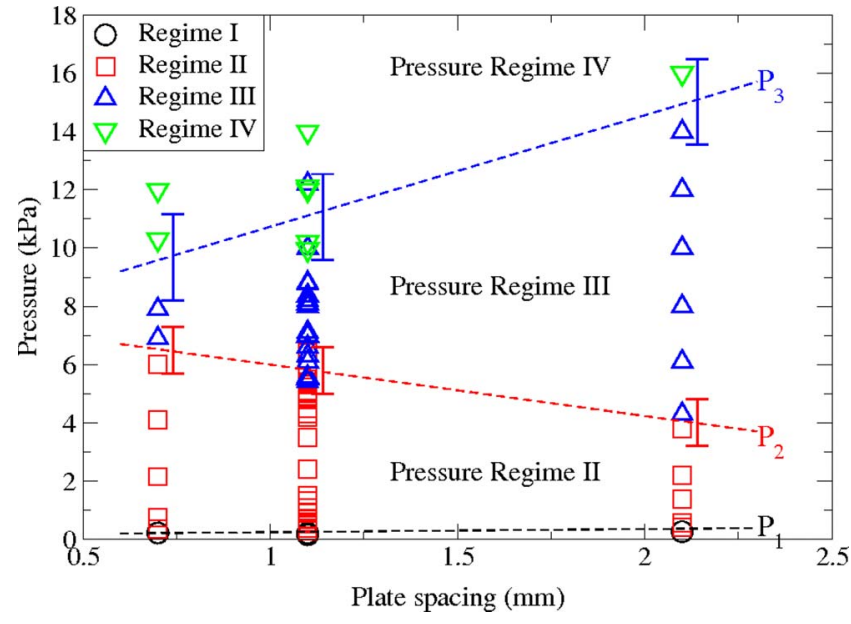

FIG. 7. (Color online) Phase diagram where we have classified and separated the patterns into pressure regimes. The patterns and into which regime they belong is a function of pressure and cell spacing. The dashed lines indicate the transition pressures $P_{1}, P_{2}$, and $P_{3}$ between the flow regimes.

$\simeq 5.9 \pm 0.5 \mathrm{kPa}$, and $P_{2}(2.1 \mathrm{~mm}) \simeq 4.0 \pm 0.5 \mathrm{kPa}$. However, the trend is the opposite for the transition between the third and fourth regime, where $P_{3}$ is an increasing function of the plate separation: $P_{3}(0.7 \mathrm{~mm}) \simeq 9.0 \pm 1.0 \mathrm{kPa}, P_{3}(1.1 \mathrm{~mm})$ $\simeq 11.0 \pm 1.0 \mathrm{kPa}$, and $P_{3}(2.1 \mathrm{~mm}) \simeq 14.0 \pm 1.0 \mathrm{kPa}$.

The transition pressures are represented by a pressure interval rather than a uniquely defined value, which is due to the fact that identical realization of the initial conditions of the granular packing from one experiment to the other is not possible. For each trial the granular packing will always present small variations in, e.g., solid fraction and disorder associated to their internal configuration.

The error bars are based on the scattering of data points from the experiments of the $d=1.1 \mathrm{~mm}$ configuration, for which the number of experiments conducted is far more significant than the other two configurations. The length of the bars is determined by the overlap of data points between successive regimes. We assume that the uncertainty is of the same order for all plate separations. For $P_{1}$ the error bars are smaller than the symbol size and therefore omitted in Fig. 7 due to presentation considerations.

A character of the flow is worth noticing in these experiments: The flow is mainly oriented along the plates, and the velocity and density seem mainly invariant along the vertical axis in the two thinnest cells. The system exhibits a "quasitwo-dimensional" (quasi-2D) character. We see, for instance, from Fig. 3 that in the 0.7 - and 1.1-mm cells, the bottom plate in the emptied zone is swept clean of grains. However, in the largest (2.1-mm-thick) cell, 14-28 grain diameters gather between the plates and the "quasi-2D" aspect starts to lose its validity. The blurred appearance for these experiments (see Fig. 3) is coupled with a more "3D-like" behavior where the edges of the finger formations have a slope at a low angle of repose, and also layers of grains are deposited on the bottom plate within the finger formation during propagation. The injected air moves the upper particles more easily. This might be due to the fact that the gas phase travels 


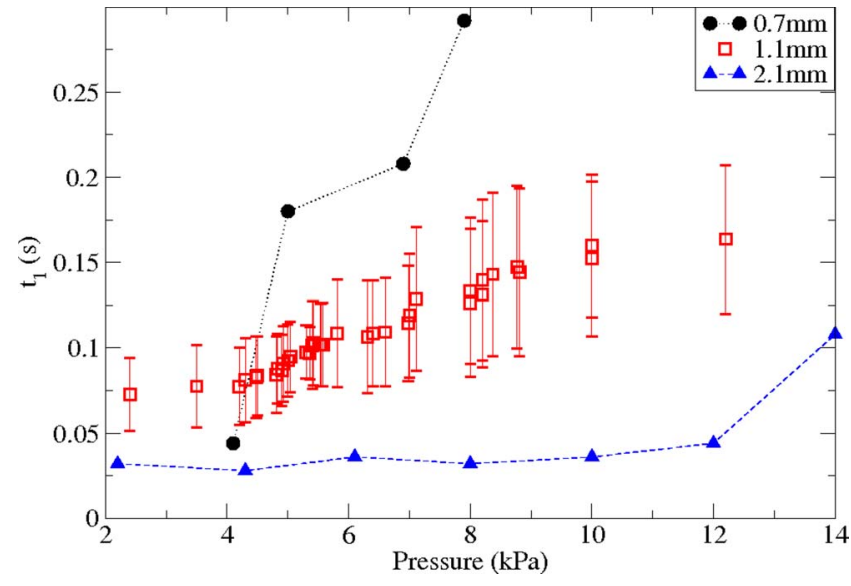

FIG. 8. (Color online) The time $t_{1}$ a as function of injection pressure for the three different plate separations. $t_{1}$ is the time between the injection start and the first stop of the growth of the emptied structure.

more favorably along the upper plate, combined with the friction between the granular material and the bottom plate being higher than along the upper plate due to the gravity acting locally on the granular column.

\section{FINGER DYNAMICS}

Properties of the pattern formations emerging during air injection, such as the characteristic times $\left(t_{1}, t_{2}\right.$, and $\left.t_{3}\right)$, position of the structure front, its width, and finger velocity, are all sensitive to changing the injection pressure and/or plate separation. These are measurements that we will now compare for different $\Delta P$ and $d$.

The fact that an identical realization of a packing from one experiment to another is impossible due to a high level of randomness and heterogeneity in the granular packing leaves us with significant scattering of data points acquired through the experiments. To determine the mean value and standard deviation and interpret the data in a conclusive and statistically reliable manner, we perform running averages on the experimental set. The mean value is obtained independently for each plate separation and gives an indication of the variations of the observables expected as a function of this parameter. The standard deviations of the observables, which are used to define the error bars, are obtained from the scattering of the data points within the interval used by the running average. The averaging is applied over the set of experiments carried out in the 1.1-mm-thick system, which is the most significant one statistically speaking. We do not expect the standard deviation of the observed quantities to vary significantly with the plate separation.

The time to form a stable structure (in pressure regime 2) or a temporarily stable structure (in pressure regime 3 ), $t_{1}$, and the length of the structure at this moment, $l\left(t_{1}\right)$, is increasing with the injection pressure, as shown, respectively, in Figs. 8 and 9. Changing the plate separation also seems to affect $t_{1}$ and $l\left(t_{1}\right)$. They both become more sensitive to variations in the injection pressure as the plate separation decreases. We cannot see the systems behaving very differently

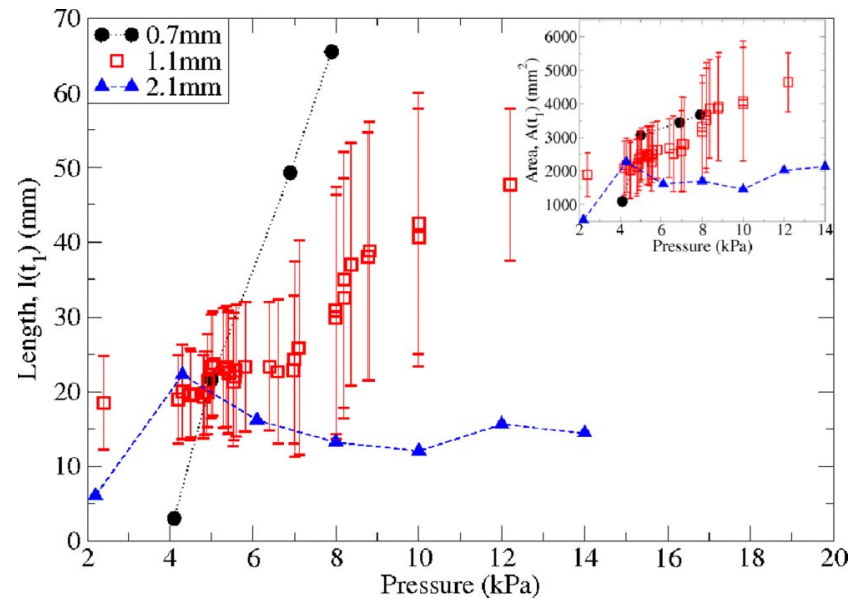

FIG. 9. (Color online) The length $l\left(t_{1}\right)$, penetration depth of the emptied structure after its initial growth, as function of pressure and plate separation.

at low and moderate injection pressures. For the area at time $t_{1}, A\left(t_{1}\right)$ (inset in Fig. 9), only the 2.1-mm case differs, while the $0.7-\mathrm{mm}$ and $1.1-\mathrm{mm}$ cases are quite comparable.

Depending on the injection pressure and the pressure regime we are in, the deformation of the outlet boundary can either be due to decompaction or compaction. The time $t_{2}$, which is the time it takes for the outlet boundary to start deforming, is seen to decrease with increasing pressure for all plate separations in Fig. 10. From Fig. 10 we conclude that varying the plate separation does not significantly influence $t_{2}$.

The time before the inner boundary starts moving again, $t_{3}$, is also not sensitive to the plate separation either, as observed from Fig. 11. $t_{3}$ is decreasing as a function of the injection pressure within the third pressure regime, from an infinite value in the first or second regime, toward zero entering the fourth pressure regime.

The velocity of the front of the emptied structure as a function of time is displayed in Fig. 12 and changes characteristics from one pressure regime to the other. Generally, the velocity $v$ increases with injection pressure. In the first pres-

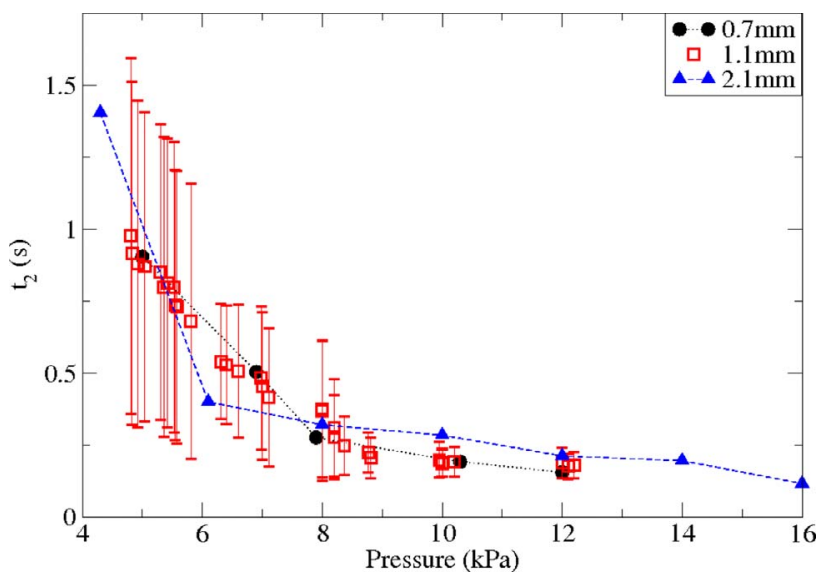

FIG. 10. (Color online) Time before deformation of the outlet grain boundary $t_{2}$. 


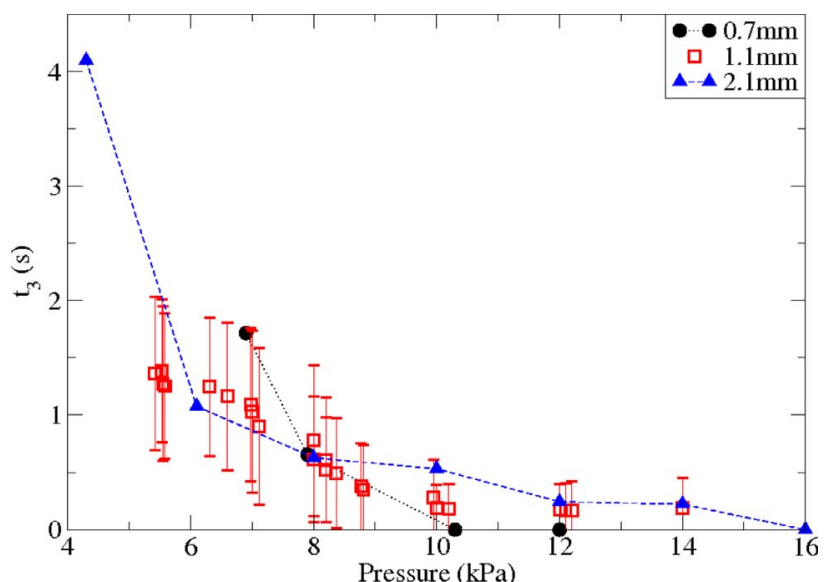

FIG. 11. (Color online) The time $t_{3}$ as a function of injection pressure for the three different plate separations. $t_{3}$ is the time elapsed between the start of the experiment and the start of the second period of motion of the inner boundary.

sure regime, $v=0$ all over. The velocity peaks during formation of an emptied structure within the second pressure regime, before the structure is stabilized after $t_{1}$ and the velocity falls to zero. For experiments within the third pressure regime, it is worth noticing that in the time interval from $t_{1}$ to $t_{3}$ where the front is more or less inactive, there are occasional stick-slip motions before a finger accelerates toward breakthrough after $t_{3}$. In practice, to discriminate this fast stick-slip motion from the more global rest period, $t_{3}$ is measured as the last moment where a zero velocity of the finger tip is observed. In the continuous-flow regime, the fourth pressure regime, the finger does not go into a rest state on its way to breakthrough, but is certainly slowed down when friction is mobilized.

To obtain a characteristic value for the velocity as function of $\Delta P$ and $d$, the average velocity of the tip of the most advanced finger is found within a central window extending over distances from the inlet going from $L / 4$ to $3 L / 4$ as presented in Fig. 13. Here $L \approx 60 \mathrm{~cm}$ is the length of the

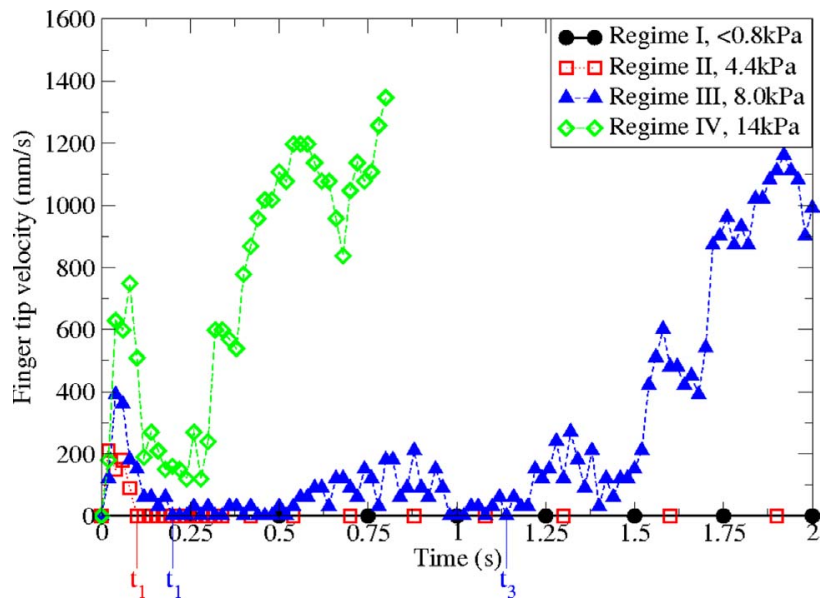

FIG. 12. (Color online) Velocity as a function of time for different injection pressures within the four defined pressure regimes.

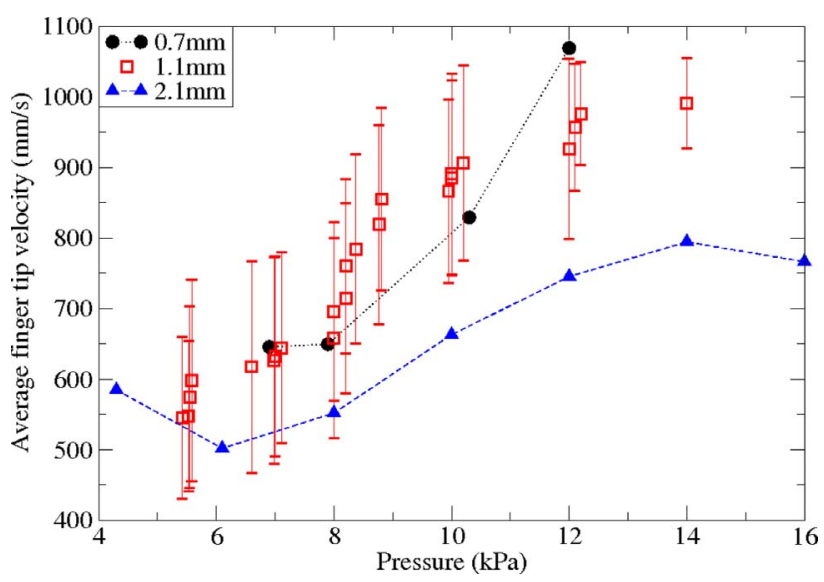

FIG. 13. (Color online) Average velocity of the most advanced finger as a function of pressure for the three different plate separations. The velocity is measured and averaged over a window at distances of the inlet from $L / 4$ to $3 L / 4$, where $L \approx 60 \mathrm{~cm}$ is the length of the granular packing before injection is initiated.

granular packing before the air injection is initiated. Since we are interested in the average speed of the progressing finger formation, it is reasonable to neglect the first stages, which include rapid growth and a transient rest stage with sporadic stick-slip motion, and consider only the continually advancing finger. When particles start to be pushed out of the cell, friction decreases, which in turn results in an acceleration of the finger-which may cause an undesirable effect on our velocity calculations also. So choosing a window from $L / 4$ to $3 L / 4$ ensures that we are averaging over a zone where the velocity is almost constant and restricted to little randomness. We can conclude that there is an obvious increase of the finger velocity as the pressure is increasing. Changing the plate separation does not seem to affect the average finger velocities significantly. The finger velocities of the $0.7-\mathrm{mm}$ cell fall within the error bars of the 1.1-mm configuration and likewise for the 2.1-mm case at low pressures. At higher pressures, the $2.1-\mathrm{mm}$ case differs slightly from the other data, but falls within two standard deviations, regarded to be within the natural scattering of data points within our system. We conclude that the plate separation chosen for these experiments does not influence significantly the finger tip velocity.

As the fingers advance toward breakthrough, the interior of the structure is slightly eroded. This can be seen as discrepancies between subsequent graphs both for the total and averaged finger width as function of position in Fig. 14. Here the average finger width is the total width of the intersection between the empty zone and cuts perpendicular to the flow direction, divided by number of fingers. For comparison and justification of the differences between the total and averaged finger width an image of the finger having developed $1 L$ is inserted into the plot.

It is also possible to measure alternatively a characteristic finger width for a structure that has advanced over a distance $L$ through the entire cell, as $\hat{w}(\Delta P, d)=A / L$, where $A$ is the area of the emptied zone. The number of fingers does not affect $\hat{w}$. This alternative measure is shown in Fig. 15. We 


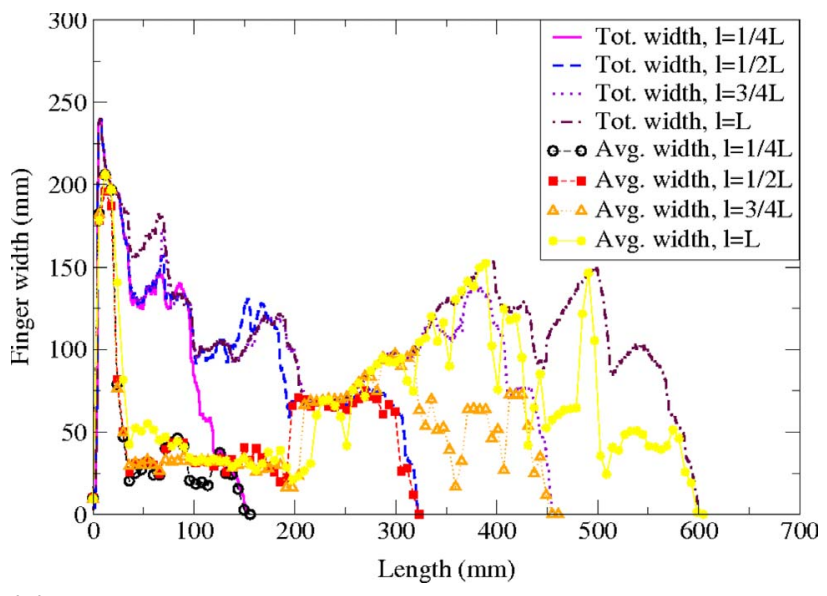

(a)

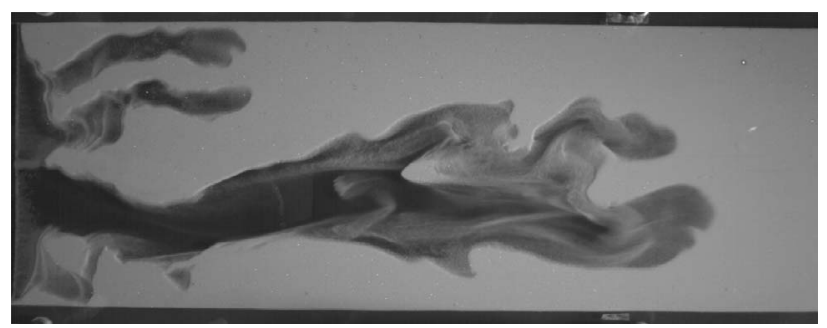

(b)

FIG. 14. (Color online) Total and average finger widths as function of time when the finger has advanced a distance $L / 4, L / 2$, $3 L / 4$, and $L$, where $L \approx 60 \mathrm{~cm}$ is the initial length of the granular packing. The experiment is performed with $1.1-\mathrm{mm}$ plate spacing at $14 \mathrm{kPa}$ overpressure. Below the graph a picture of a finger that has advanced a distance $l=L$ is inserted to illustrate and help the comparison between total and averaged finger widths.

notice a trend of fingers widening with respect to increasing pressure, while the plate separation does not seem to be of any influence.

\section{DISCUSSION}

The origin of the instability is similar to the mechanism first described by Saffman and Taylor [18], in nonmiscible fluid flow where a low-viscous fluid displaces a more viscous one. The pressure gradient $\vec{\nabla} P$ is the driving force of the granular motion: the fluid drag over the grains is a growing function of the local velocity of the air through the grains, which is obtained by dividing the Darcy velocity [59] by the local porosity $\Phi$, giving [61]

$$
\vec{u}_{a}=-\frac{\kappa(\Phi)}{\eta \Phi} \vec{\nabla} P
$$

where $\eta$ is the air viscosity and $\kappa(\Phi)$ the local porositydependent permeability of the granular assembly. Within the fingers the permeability is huge compared to the one in the pore space of the granular material, which will favor air flow in the emptied region. Consequently, the pressure is more or less constant within the emptied structure, and at the tip of

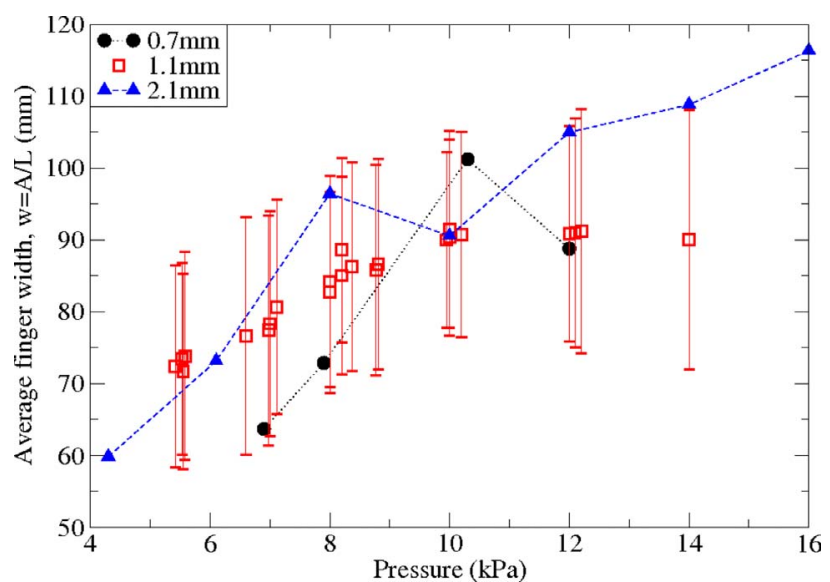

FIG. 15. (Color online) Average finger widths $w=A / L$, as a function of pressure when a finger has advanced a distance equal to the initial length $L \approx 60 \mathrm{~cm}$.

the longest finger, the distance $l$ from the tip to the open boundary is the smallest, so that the driving pressure gradient $\vec{\nabla} P \simeq \Delta P / l$ is largest here. Growth of the most advanced finger is therefore favored at the expense of the rest of the structure. This is the origin of the observed instability, which gives rise to fingering and destabilizes the initially straight interfaces. In contrast with the Saffman-Taylor instability observed in nonmiscible fluid flow, the pure air and the granular/gaseous fluid mixture exchange mass, i.e., interpenetrate without any surface tension effect. The stabilizing phenomenon is not surface tension here, but the friction mobilized between the granular matrix and the confining plates.

Another mechanism that contributes to the stabilization of the structure front is a result of another important difference between the system described here and Saffman and Taylor's system: in the present case, the granular material and the fluid are interpenetrating. As the air flows through the granular material, the pressure field diffuses and the gradient will smoothen out and be reduced over the front. If the growing friction becomes large enough to balance the decreasing hydrodynamic drag on the particles, the emptied structure stops growing.

In the formation of the emptied structure and the associated compacted region the initial solid fraction may be of great importance. For an initially loose realization of the granular packing, as presented in this study, the material must compact before "jamming" through the mobilized friction and balance with the hydrodynamic drag force can occur. Before the onset of jamming particles may be displaced over a large extent, leaving what is defined as an emptied structure. For higher initial packing fraction obtained by, e.g., tapping the cell, the material is precompacted and the friction acting between the granular material and the plates is higher due to stronger arching effects. Further compaction and particle displacement become more difficult, and emptied structures of smaller extent are expected. No systematic experimental studies have been conducted regarding the effect of initial packing fraction variations on the extension of the emptied and compacted region for this system, but it is evident in numerical simulations performed in relation with 


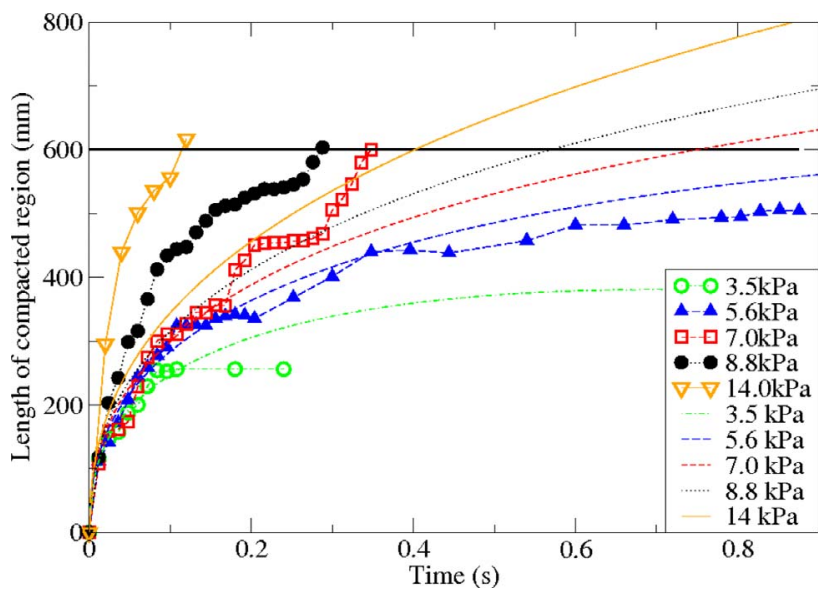

FIG. 16. (Color online) Position of the compacted zone's most advanced point as a function of time at different injection pressures. The plate separation is $1.1 \mathrm{~mm}$. The analytical solution in Eq. (12) gives a good fit for low injection pressures. Friction coefficient $\mu$ $=0.20$ is used for this particular fit.

the studies on granular flow in the circular cell geometry in Ref. [14].

As the emptied structure grows, the material ahead of this structure compacts over a depth dependent on the size of the structure. Information on the size of the compacted region is extracted using image subtraction techniques. In Fig. 16, the position $x$ of the most advanced point of the compacted zone is plotted as function of time $t, x\left(P_{i}, t\right)$, for five experiments at different injection pressures $P_{i}$, in the 1.1-mm-thick cell: one within the second pressure regime, three within the third, and one from the fourth. For the lowest injection pressures, the empty structure and its associated compacted structure reach saturation after $\sim 0.1 \mathrm{~s}$ and no deformation of the outlet boundary is seen. For larger injection pressures $P_{i}$, the growth of this compacted zone eventually reaches the outer boundary at a time previously defined as $t_{2}\left(P_{i}\right)$.

We propose below a simple model which in the lowestpressure regimes successfully renders for these two observables the extent of the compacted zone as function of time, $x\left(P_{i}, t\right)$, and the time to deform the outer boundary, $t_{2}\left(P_{i}\right)$.

Following from the mass conservation of air,

$$
\frac{\partial \rho_{a}}{\partial t}+\vec{\nabla} \cdot\left[\rho_{a} \vec{u}_{a}\right]=0
$$

where $\rho_{a}$ is the mass of air per unit of total volume (including grains and pores) and $\vec{u}_{a}$ is the interstitial gas velocity.

Considering air as an isothermal ideal gas,

$$
\rho_{a}=\frac{\Phi P}{k_{B} T} M,
$$

where $M$ is the mean mass of the gas molecules, $k_{B} T$ is Boltzmann's constant times the temperature, $P=P(x, t)$ is the position- and time-dependent pressure within the granular material, and $\Phi$ is the porosity of the granular matrix.
During the initial compaction stage, the velocity of the granular medium is negligible compared to the gas velocity, so that the velocity of the interstitial gas is directly obtained from Eq. (2). Neglecting the small initial variations of porosity, $\Phi$ is constant and homogeneous so that

$$
\Phi \frac{\partial P}{\partial t}=\vec{\nabla} \cdot\left[\frac{\kappa}{\eta} P \vec{\nabla} P\right]=0 .
$$

Writing $P=P_{0}+\Delta P$, with $P_{0}$ the atmospheric pressure at the outlet, the imposed overpressure is only a few percent of the atmospheric pressure, so that $\Delta P \ll P_{0}$, and Eq. (5) can be approximated as a simple diffusion law

$$
\frac{\partial P}{\partial t}=D \nabla^{2} P
$$

with a diffusion constant $D=\kappa P_{0} / \eta \Phi$. The atmospheric pressure is $P_{0}=10^{5} \mathrm{~Pa}$, and the viscosity of air $\eta=1.8$ $\times 10^{-5}$ Pa s. With the porosity $\Phi=1-\rho_{s} \approx 0.45$ for the loose-packed granular "plug," the permeability can be estimated using the density dependent Carman-Kozeny relation [62], so that for particles of mean radius $a \approx 50 \mu \mathrm{m}$,

$$
\kappa=\frac{a^{2}}{45} \frac{\phi^{3}}{(1-\phi)^{2}}=1.67 \times 10^{-11} \mathrm{~m}^{2} .
$$

The diffusion constant can then be estimated as $D$ $\approx \kappa P_{0} / \eta \Phi=0.207 \mathrm{~m}^{2} \mathrm{~s}^{-1}$. A characteristic time for the overpressure to diffuse through the length of the granular plug $L=0.60 \mathrm{~m}$ is

$$
\tau=\frac{L^{2}}{2 D} \simeq 0.9 \mathrm{~s},
$$

which is a rough estimate, but in agreement with what is observed for low pressures in Figs. 10 and 17.

We impose a constant overpressure at the inlet at positive times and a null one at negative ones, i.e., $\Delta P(x=0, t)$ $=P_{i} \Theta(t)$ where $\Theta$ is a Heaviside function, while the overpressure is maintained at zero at the outlet. As long as the packing stays essentially immobile, the pressure source is obtained by solving this diffusion equation with these boundary conditions, with initial condition $P(x \geq 0, t=0)=0$. A last approximation can be done by considering a long packing, i.e., by considering that the outlet condition is imposed at infinite distances: $P(x=\infty, t)=0$. This leads to a simple analytical solution of the diffusion equation,

$$
\Delta P(x, t)=P_{i} \frac{2}{\sqrt{\pi}} \int_{x / \sqrt{2 D t}}^{\infty} e^{-y^{2}} d y .
$$

The pressure gradient $\vec{\nabla} P=\partial P / \partial x$ gives the fluid velocity $\vec{u}_{a}=-(\kappa / \eta \Phi) \vec{\nabla} P$ which we use to estimate the Reynolds number $\operatorname{Re}=\rho_{\text {air }} u_{a} a / \eta$. The mass density of air is $\rho_{\text {air }}$ $=1.1 \mathrm{~kg} \mathrm{~m}^{-3}$. The pressure gradient and hence the velocity are at their highest at the inlet boundary $x=0$, which gives 


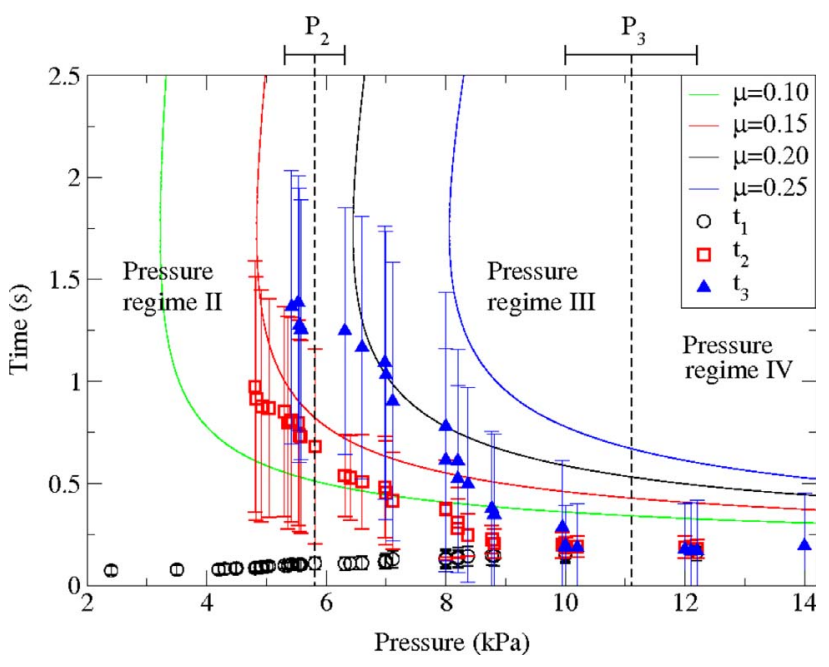

FIG. 17. (Color online) Time to form an initial emptied structure, $t_{1}$; time before deformation of the boundary, $t_{2}$; and time before breakthrough, $t_{3}$, as a function of injection pressure. $t_{2}$ is compared with the analytical solution for different friction coefficients $\mu$. Pressure regimes and their associated thresholds with estimated error bars are indicated.

$$
\mathrm{Re}_{\max } \approx 1.1 \times 10^{-5} \mathrm{~s}^{1 / 2} \mathrm{~Pa}^{-1} \frac{P_{i}}{\sqrt{t}} .
$$

Such a small Reynolds number confirms that we are operating within the laminar flow regime for our experiments and justifies the use of the Darcy law in our theoretical model.

One can exploit the diffusion equation (9) to estimate the extent of the compacted zone: At the front of the mobile zone, the initial in-plane forces are presumably negligible, and we can assume that the motion of the grains is caused by the fact that the fluid drag exceeds the friction threshold exerted by the plates over the grains, at the tip of the mobile zone. For a layer of thickness $\Delta z$ the fluid drag per unit area of the plates is $-\vec{\nabla} P \Delta z$. The friction can be modeled as a Coulomb friction, with a pressure over the bottom plate obtained from the weight of the grain column and forces neglected along the top plate, so that the friction force per unit area is $f=\mu(1-\Phi) \rho g \Delta z$, where $\mu$ is a Coulomb friction coefficient and $\rho=2.6 \mathrm{~g} \mathrm{~cm}^{-3}$ is the density of the glass beads. Using the pressure profile, Eq. (9), this leads to the following for the position of the tip of the mobile zone:

$$
\mu(1-\Phi) \rho g=\sqrt{\frac{2}{\pi D t}} P_{i} e^{-x^{2} / 2 D t} .
$$

Solving this equation for $x=x(t)$ gives the position beyond which grains will not have moved:

$$
x(t)=\sqrt{2 D t} \sqrt{-\min \left[\ln \left(\sqrt{\frac{\pi D t}{2}} \frac{\mu(1-\Phi) \rho g}{P_{i}}\right), 0\right]} .
$$

Note that for small times the logarithm function is negative so that the square root is a real number. The function $x(t)$ is positive and increasing up to a maximum value

$$
x_{m}=\frac{P_{i}}{\mu(1-\Phi) \rho g \sqrt{\pi e / 2}},
$$

which occurs at a time

$$
t_{m}=\frac{2 P_{i}^{2}}{\pi e D[\mu(1-\Phi) \rho g]^{2}} .
$$

When $t>t_{m}$ the coordinate $x(t)$ decreases through positions where displacement has already occurred and where nothing more will happen. Eventually $x(t)=0$ and for larger times, the pressure force is smaller than the friction threshold everywhere.

Using $\mu=0.20$, close to what was estimated in simulations of a similar system [14], the analytical solution, Eq. (12), gives a good approximation for the position of the tip of the mobile zone at low and moderate injection pressures (within the second pressure regime and beginning of the third), as seen when comparing with experimental data in Fig. 16. This is valid up to the moment when it reaches the outer boundary.

This solution fails, however, to give a description at higher injection pressures where the material is observed to compact all the way up to the outlet boundary in roughly $\sim 0.1-0.3$ s (see Fig. 16). The growth process of the compacted region is much quicker than the time it takes the air flow to travel the linear distance of the system size. In this case, the assumption that contact forces between the particles at the tip of the compacted zone are negligible probably fails, and presumably the compaction is carried through particle contact rather than through hydrodynamic drag.

Eventually, this solution can be considered to derive the time the moment when the fluid drag is sufficient to deform the outer boundary, which defined the time $t_{2}$. Specifying $x\left(t_{2}, P_{i}\right)=L$ in Eq. (12), we obtain an implicit equation for $t_{2}\left(P_{i}\right)$. In Fig. 17 all the pressure-dependent time measurements $t_{1}, t_{2}$, and $t_{3}$ for the experimental sets of the 1.1-mm configuration are compiled together for comparison. The pressure regimes and their associated transition pressures with estimated error bars are also indicated. The analytical solution for $t_{2}$ is plotted for different friction coefficients $\mu$, where $\mu=0.15$ fit the experiments very closely. This value of $\mu$ deviates slightly from the one used to model the compacted region-Eq. (12) - in Fig. 16. The foundation of the model of both the compacted region and the time before deformation of the outlet boundary is the solution in Eq. (9) for an infinite length of the conducting medium, while in the real world the packing is indeed of finite extension. This, together with the fact that porosity variations are neglected, might be a significant source of error. In conclusion, we estimate roughly $\mu \sim 0.15-0.20$.

Local porosity variations may modify this simplified model. Its influence can be estimated through comparing the pressure gradients $\vec{\nabla} P_{\Phi}(x, t)$ and $\vec{\nabla} P_{\Phi_{c}}(x, t)$, found by $\vec{\nabla} P(x, t)=\partial P / \partial x$ of Eq. (9), respectively, for the initial solid fraction $\Phi=1-\rho_{s}^{\text {init }}=0.45$ and the compacted state represented by $\Phi_{c}=1-\rho_{s}^{c o m p}=0.42$. By division 
$\vec{\nabla} P_{\Phi_{c}}(x, t) / \vec{\nabla} P_{\Phi}(x, t)$ in the limit of small $x$ and $t$-i.e., near the inlet air/grain interface during the first stage of displacement and compaction-we find that $\vec{\nabla} P_{\Phi_{c}}$ may be $\sim 16 \%$ higher than $\vec{\nabla} P_{\Phi}$. Local porosity variations may indeed induce large pressure variations and significantly influence the grain dynamics.

\section{SUMMARY AND CONCLUSIONS}

Through our study we have given a qualitative and quantitative description of displacement patterns in granular materials confined in a rectangular Hele-Shaw cell.

The driving force in this system is the pressure gradient acting between the front of the pattern formation and the outlet grain boundary. The competing stabilizing mechanisms are friction mobilized between the granular material and the confining plates. These two mechanisms can be controlled by tuning the injection pressure and separation between the glass plates, giving a wide range of patterns. Given the different pattern formations and their dynamic behavior, four pressure regimes are defined into which the patterns are categorized.

In the first pressure regime there is no grain motion and the air simply permeates through the pores of the granular packing.

In the second pressure regime, particles are being displaced, forming a stable formation after $t_{1}$ seconds. The patterns appear with a relatively flat interface for large plate separation and become increasingly bulged when the plate separation is decreased. Deformation of the pattern front is more noticeable when a higher injection pressure is applied. The time to form a pattern, $t_{1}$, is increasing with the injection pressure $\Delta P$ and smaller plate spacing, which is also the case of the pattern size $l\left(t_{1}\right)$. The extent of the compacted zone is also increasing with $\Delta P$. This feature is also captured in our theoretical model based on balance of the hydrodynamic forces acting on the particles (governed by the diffusion equation) and Coulomb friction between the particle matrix and confining plates.

The instability becomes more pronounced when entering the third pressure regime - especially for narrow plate separation. An empty structure is established in $t_{1}$ seconds before a transient stabilization. Deformation of the outlet boundary is observed after a $t_{2}$ seconds. The material near the bound- ary is locally decompacted after the propagation of a diffusive pressure front, which decreases friction with the confining plates and the ability to balance the hydrodynamic forces. The granular material is locally fluidized during this decompaction, eventually resulting in a finger breaking through the sample. A secondary motion of the inner boundary happens after $t_{3}$ seconds. $t_{3}$ is pressure dependent, but not sensitive to plate separation. The duration of the rest-state, $t_{3}-t_{1}$, becomes shorter with higher injection pressure and approaches zero. $t_{3}-t_{1}=0$ marks the transition to the fourth pressure regime. Close to this transition, but still in the third regime, deformation of the outlet boundary after $t_{2}$ seconds is due to the compacted zone reaching the boundary carried through particle contacts, rather than being local decompaction as a result of hydrodynamic forces acting on the outermost particles. Our prediction of the length of the compacted zone fails in this case, since it is based on hydrodynamical considerations.

When a continually advancing finger is observed from beginning until the end, the system is classified in a fourth pressure regime. The fluid and grain flow are strongly coupled and instable.

Generally for the pressure regimes the characteristic times $t_{1}, t_{2}$, and $t_{3}$, when defined within the regime, are all pressure dependent. While $t_{2}$ and $t_{3}$ are decreasing with $\Delta P$ and do not show a dependence on plate separation, $t_{1}$ is increasing with both $\Delta P$ and plate spacing $d$. Our theoretical model gives a good fit for $t_{2}$ in the range of pressures where the advancement of the compacted region is not dominated by solid contacts.

Features of the propagating finger, such as the tip velocity and width, are increasing with higher injection pressure, but are not sensitive to plate separation.

Although no systematic studies on the influence of varying the initial solid fraction have been conducted, it might be of central importance to the grain dynamics both locally and globally.

\section{ACKNOWLEDGMENTS}

This work was supported by NFR, the Norwegian Research Council, through a Petromax and a SUP grant, and by CNRS through a PICS grant. We thank Anke Lindner, Christophe Chevalier, and Ernesto Altshuler for many interesting and helpful discussions.
[1] J. F. Davidson and D. Harrison, Fluidization (Academic Press, New York, 1971).

[2] J. F. Davidson, Bubbles in Fluidized Beds (Kluwer Academic, New York, 1995), p. 197.

[3] K. S. Lim, J. X. Zhu, and J. R. Grace, Int. J. Multiphase Flow 21, 141 (1995).

[4] S. McNamara, E. G. Flekkøy, and K. J. Måløy, Phys. Rev. E 61, 4054 (2000).

[5] G. W. Baxter, R. P. Behringer, T. Fagert, and G. A. Johnson, Phys. Rev. Lett. 62, 2825 (1989).
[6] K. J. Måløy, T. L. Pennec, E. G. Flekkøy, D. Bideau, M. Ammi, J. C. Messager, X. L. Wu, and A. Hansen (unpublished).

[7] T. Le Pennec, K. J. Måløy, E. G. Flekkøy, J. C. Messager, and M. Ammi, Phys. Fluids 10, 3072 (1998).

[8] E. G. Flekkøy and K. J. Måløy, Phys. Rev. E 57, 6962 (1998).

[9] D. Gendron, H. Troadec, K. J. Måløy, and E. G. Flekkøy, Phys. Rev. E 64, 021509 (2001).

[10] E. G. Flekkøy, S. McNamara, K. J. Måløy, and D. Gendron, Phys. Rev. Lett. 87, 134302 (2001). 
[11] X.-1. Wu, K. J. Måløy, A. Hansen, M. Ammi, and D. Bideau, Phys. Rev. Lett. 71, 1363 (1993).

[12] T. LePennec, K. J. Måløy, A. Hansen, M. Ammi, D. Bideau, and X. L. Wu, Phys. Rev. E 53, 2257 (1996).

[13] E. Lemaire, Y. O. M. Abdelhaye, J. Larue, R. Benoit, P. Levitz, and H. VanDamme, Fractals 1, 968 (1993).

[14] Ø. Johnsen, R. Toussaint, K. J. Måløy, and E. G. Flekkøy, Phys. Rev. E 74, 011301 (2006).

[15] P. Mills, P. Cerasi, and S. Fautrat, Europhys. Lett. 29, 215 (1995).

[16] P. Cerasi and P. Mills, Phys. Rev. E 58, 6051 (1998).

[17] H. S. Hele-Shaw, Nature (London) 58, 34 (1898).

[18] P. G. Saffman and G. I. Taylor, Proc. R. Soc. London, Ser. A 245, 312 (1958).

[19] D. Bensimon, L. P. Kadanoff, S. Liang, B. I. Shraiman, and C. Tang, Rev. Mod. Phys. 58, 977 (1986).

[20] R. L. Chuoke, P. van Meurs, and C. van der Poel, Trans. AIME 216, 188 (1959).

[21] J. V. Maher, Phys. Rev. Lett. 54, 1498 (1985).

[22] A. Lindner, D. Bonn, E. C. Poiré, M. B. Amar, and J. Meunier, J. Fluid Mech. 469, 237 (2002).

[23] D. Bonn, H. Kellay, M. Ben Amar, and J. Meunier, Phys. Rev. Lett. 75, 2132 (1995).

[24] G. Daccord, J. Nittmann, and H. E. Stanley, Phys. Rev. Lett. 56, 336 (1986).

[25] J. Nittmann, G. Daccord, and H. E. Stanley, Nature (London) 314, 141 (1985).

[26] E. Lemaire, P. Levitz, G. Daccord, and H. Van Damme, Phys. Rev. Lett. 67, 2009 (1991).

[27] H. Zhao and J. V. Maher, Phys. Rev. E 47, 4278 (1993).

[28] T. Hirata, Phys. Rev. E 57, 1772 (1998).

[29] R. Chandler, J. Koplik, K. Lerman, and J. F. Willemsen, J. Fluid Mech. 119, 249 (1982).

[30] R. Lenormand and C. Zarcone, Phys. Rev. Lett. 54, 2226 (1985).

[31] R. Lenormand and C. Zarcone, Transp. Porous Media 4, 599 (1989).

[32] G. M. Homsy, Annu. Rev. Fluid Mech. 19, 271 (1987).

[33] K. J. Måløy, J. Feder, F. Boger, and T. Jøssang, Phys. Rev. Lett. 61, 2925 (1988).

[34] O. I. Frette, K. J. Maløy, J. Schmittbuhl, and A. Hansen, Phys. Rev. E 55, 2969 (1997).

[35] E. Aker, K. J. Måløy, and A. Hansen, Phys. Rev. Lett. 84, 4589 (2000).

[36] G. Løvoll, Y. Méheust, R. Toussaint, J. Schmittbuhl, and K. J. Måløy, Phys. Rev. E 70, 026301 (2004).
[37] R. Toussaint, G. Løvoll, Y. Méheust, K. J. Måløy, and J. Schmittbuhl, Europhys. Lett. 71, 583 (2005).

[38] T. A. Witten and L. M. Sander, Phys. Rev. Lett. 47, 1400 (1981).

[39] T. A. Witten and L. M. Sander, Phys. Rev. B 27, 5686 (1983).

[40] P. Meakin, Phys. Rev. A 27, 1495 (1983).

[41] L. Paterson, Phys. Rev. Lett. 52, 1621 (1984).

[42] E. L. Hinrichsen, K. J. Måløy, J. Feder, and T. Jøssang, J. Phys. A 22, L271 (1989).

[43] F. A. L. Dullien, Chem. Eng. Technol. 11, 407 (1988).

[44] J. Bear, Dynamics of Fluids in Porous Media (Elsevier, New York, 1972).

[45] M. Sahimi, Rev. Mod. Phys. 65, 1393 (1993).

[46] P. van Meurs, Trans. AIME 210, 295 (1957).

[47] A. K. Gustensen and D. H. Rothman, J. Geophys. Res. 98, 6431 (1993).

[48] H. A. Tchelepi and F. M. Orr, SPE Reservoir Eng. 9, 266 (1994).

[49] I. Ginzbourg and P. M. Adler, Transp. Porous Media 20, 37 (1995).

[50] D. Mader, Hydraulic Proppant Fracturing and Gravel Packing (Elsevier, Amsterdam, 1989).

[51] Y. Gordeyev, Int. J. Rock Mech. Min. Sci. 30, 233 (1993).

[52] P. Valkø and M. J. Econimedes, Hydraulic Fracture Mechanics (Wiley, New York, 1995).

[53] E. Fjaer, R. M. Holt, P. Horsrud, A. M. Raaen, and R. Risnes, Petroleum Related Rock Mechanics (Elsevier, Amsterdam, 1992).

[54] W. S. Fyfe, N. Price, and A. B. Thompson, Fluids in the Earth's Crust (Elsevier, Amsterdam, 1978).

[55] A. Levermann and I. Procaccia, Phys. Rev. Lett. 89, 234501 (2002).

[56] E. G. Flekkøy, A. Malthe-Sørenssen, and B. Jamtveit, J. Geophys. Res. 107, 2151 (2002).

[57] F. Tzschichholz, H. J. Herrmann, H. E. Roman, and M. Pfuff, Phys. Rev. B 49, 7056 (1994).

[58] F. Tzschichholz and H. J. Herrmann, Phys. Rev. E 51, 1961 (1995)

[59] H. Darcy, Les Fontaines Publiques de la Ville de Dijon (Dalmont, Paris, 1856).

[60] H. A. Janssen, Z. Vereines Deutscher Ingenieure 39, 1045 (1892).

[61] D.-V. Anghel, M. Strauss, S. McNamara, E. G. Flekkøy, and K. J. Måløy, Phys. Rev. E 74, 029906(E) (2006).

[62] P. Carman, Trans. Inst. Chem. Eng. 15, 150 (1937). 